Fecha de recepción: abril 2020 Fecha de aceptación: mayo 2020 Versión final: junio 2020

\section{Fronteras flexibles del diseño. Experiencias de diseño colaborativo en el sector textil marplatense}

María Celina Monacchi ${ }^{(1)}$

\begin{abstract}
Resumen: El diseño se manifiesta en las fronteras flexibles de la adaptación de la comunidad al cambio tecnológico. Es así como las soluciones estandarizadas no aplican igual en todos los territorios y el diseño puede contribuir como interfaz de asimilación. Este escrito narra una experiencia colaborativa con actores de la cadena textil e indumentaria de Mar del Plata en el año 2019. En un contexto complejo, las empresas, el Estado y la Universidad se unen para conformar un clúster. El diseño acompaña la construcción de su identidad a través de herramientas de Design Thinking, que acercan un marco metodológico a nuevos grupos sociales. Los resultados buscan demostrar que el aprendizaje recíproco fomenta la formación de redes que motorizan innovaciones arraigadas al territorio.
\end{abstract}

Palabras claves: Diseño colaborativo - Design Thinking - Pensamiento de diseño - Clúster local - Cadena de valor textil e indumentaria.

[Resúmenes en inglés y portugués en la página 89]

(1) Diseñadora industrial graduada de la Universidad Nacional de Mar del Plata (UNMdP). Ayudante graduado en la cátedra Taller Vertical Diseño Textil, de la carrera de Diseño Industrial de la UNMdP. Integrante desde el 2015 al Grupo de Investigación en Diseño Sustentable (GIDSu), perteneciente al Centro de Investigaciones Proyectuales y Acciones de Diseño Industrial (CIPADI) de la UNMdP. Maestranda en la Maestría en Diseño orientada a la Estrategia y la Gestión de la Innovación de la Universidad del Noroeste de Buenos Aires (UNNOBA).

\title{
Introducción
}

El presente artículo surge de un trabajo de investigación más extenso, el cual propone analizar el sector textil-indumentaria de Mar del Plata bajo la óptica del diseño, como factor estratégico de la cadena de valor. Ante la dificultad de alcanzar datos cuantitativos precisos (se refiere a un sector industrial sumamente informal y desarticulado) y la necesidad de comprender desde una mirada cualitativa esta industria y sus problemáticas, se recurrió a las herramientas de Design Thinking como metodología para trabajar de forma colabora- 
tiva con las empresas. Esta propuesta tenía dos intenciones: en primer lugar, entender de primera mano la realidad sectorial y estructurar su cadena de valor, para poder plantear estrategias de diseño que la reposicionen. En segundo término, sociabilizar respecto al rol estratégico del diseño y su posible aporte más allá del enfoque productivo al que siempre está asociado en el sector textil local.

La implementación del Design Thinking ha sido la excusa que permitió acercarse desde la disciplina a nuevos espacios sociales. Es sabido que el renombre que este concepto ha adquirido en el último tiempo, principalmente en el mundo de los negocios, generó controversia en el ámbito del diseño porque básicamente define la forma de trabajar de los diseñadores. Su base teórica no es novedosa (Simon, 1969; Rowe, 1987; Buchanan, 1992), pero sí la visibilidad que ha adquirido en el mundo empresarial y su réplica como modelo de resolución de problemas en organizaciones. La elección de este marco permite además evaluar si, en términos de desarrollo local y territorialidad, responde a condiciones corporativistas o puede aplicarse como herramienta para el conglomerado $\mathrm{PyME}^{1}$. Se puede resumir en que el fundamento es entender cómo el diseño puede insertarse como modelo de gestión estratégica en contextos locales, entendiendo sus lógicas particulares y evitando la importación de metodologías foráneas poco semejables a sus realidades.

Acompaña este enfoque, el concepto de desarrollo local: el valor del territorio como espacio de interacciones (Garbarini, 2019) y la importancia de la historia colectiva (Arocena, 2002) como movilizador a la acción. El diseño no es en absoluto ajeno a esta cuestión; Galán (2011) destaca el valor de sus profesionales como agentes de desarrollo social, donde el espacio proyectual funciona como "dispositivo articulador de recursos, formado de consensos y productor de sentido en el marco del desarrollo de las comunidades locales". Esta concepción excede ampliamente la asociación del diseño únicamente al mundo objetual y a la producción, y lo traslada a una visión sistémica. Es allí, en la propia experiencia, donde el diseño puede crear valor, desde una mirada empática que prioriza lo local, la sustentabilidad del territorio y de sus actores.

En consonancia a estas cuestiones, el artículo pretende describir una experiencia de Design Thinking realizada durante el 2019 con distintos actores del sector local, en el marco del Conglomerado Textil-Indumentaria de Mar del Plata ${ }^{2}$. Esta organización, formalizada en 2017, nuclea no sólo empresas, sino también integrantes de distintas áreas de la Universidad Nacional de Mar del Plata y del gobierno municipal, con el objetivo de reposicionar al sector en términos de innovación, tecnología, mercados, diseño, sustentabilidad, entre otros. Esta propuesta estratégica busca superar las diferencias de las cámaras empresariales y sumar otros actores a la mesa sectorial. El escenario resultó propicio para la realización de actividades colaborativas estructuradas según herramientas de diseño cualitativas. Los resultados se exponen en este artículo, teniendo en cuenta los datos cualitativos que se pudieron recabar, las problemáticas que suscitaron las herramientas y el rol del diseño en el desarrollo de esta experiencia conjunta. 


\section{Materiales y métodos}

La metodología de investigación de este trabajo debe dividirse en dos partes. Por un lado, la construcción del marco teórico para entender el objeto de estudio, la cadena de valor textil-indumentaria Mar del Plata. Esta primer parte se centra en datos cualitativos, entrecruzados con otros cuantitativos, obtenidos de fuentes bibliográficas, entrevistas no estructuradas a informantes calificados y participación en mesas sectoriales de trabajo y fuentes de archivo de diversas organizaciones afines.

Por el otro lado, el trabajo de campo se diseñó en función de la metodología que plantea el Design Thinking (Brown, 2009; Martin, 2009; Hasso-Plattner Institute, 2018), seleccionando aquellas herramientas más propicias para el caso. Como se mencionó, el trabajo tiene como inquietud la aplicación de herramientas de diseño para experiencias colaborativas y el análisis de las problemáticas del sector. Se considera que el enfoque de Design Thinking permite abordar la temática desde una visión empática y generar ideas innovadoras que colaboren con el posicionamiento de la actividad (Vianna et.al, 2016). A partir de los principios metodológicos del Design Thinking, se ha decidido realizar una readaptación de algunas de sus herramientas para la aplicación en el caso práctico de la industria textil y confeccionista de Mar del Plata. Esta modificación no apela a cambiar sustancialmente las herramientas en sí, sino que pretende guiar mejor su aplicación en el contexto enunciado anteriormente.

\section{Caracterización del sector textil-indumentaria Mar del Plata}

La población corresponde a las PyMES del sector textil-indumentaria de la ciudad de Mar del Plata. Este grupo se compone de dos sub-ramas: la industria textil (productos de tejido de punto comúnmente conocidos como sweaters) y la actividad vinculada a la indumentaria. Si bien pertenecen a un mismo sector industrial, en la escala local presentan algunas diferencias. La industria del tejido de punto tiene un peso muy marcado en la ciudad. Sus inicios datan de los años '50, asociados a las oleadas inmigratorias y el asentamiento de estas familias en la ciudad (Favero, 2011). El crecimiento de Mar del Plata como epicentro turístico nacional, provocó un fuerte desarrollo de esta actividad, acompañado por tecnificación, especialización y generación de otras actividades económicas vinculadas a este sector (Monacchi, 2017). La presencia del tejido de punto asociado a la ciudad, se consagra con su nombramiento como Capital Nacional del Pulóver, en la década del '70. Sin embargo, con la apertura de las importaciones, el producto local no pudo competir con el importado y la industria se contrajo, produciendo el cierre de numerosas fábricas, entre ellas algunas que habían sido emblemáticas de la ciudad. Se puede decir que desde el año 2000, este sector económico fluctúa entre momentos de mejora y posterior decaimiento, sin poder alcanzar una estabilidad en el tiempo. En la actualidad, el sector textil local se manifiesta en: unidades de producción descentralizadas (fasones, talleres), fuerte circulación de conocimiento en el territorio, marcado carácter paternalista y familiar en 
las empresas locales, altas tasas de informalidad laboral, reconocimiento del producto por su calidad a nivel nacional, entre otros.

En relación a la sub-rama indumentaria, ha estado en sus inicios ligado al sector del tejido de punto. Las actividades de confección se abocaban al sweater principalmente, que era el producto con mayor circulación. Cabe diferenciar que este tipo de prenda tejida tiene procesos productivos específicos que no se repiten en otro tipo de artículos de indumentaria, pero aun así permitió la incursión en las confecciones de tipo industrial. Sin embargo, a través del tiempo, estas dos sub-ramas (textil e indumentaria) comenzaron a diferenciarse. La producción de indumentaria en la ciudad comienza a notar presencia recién a fines de la década del ' 70 y ' 80 , con algunas fábricas relevantes con más de 50 ocupados (Gennero de Rearte, 2008). El sector crece hasta inicios de los años '90, sobre una estructura de pequeñas empresas, pero nunca alcanza más de dos empresas con más de 50 empleados (Gennero et al., 1991). Varias de las firmas radicadas en Mar del Plata se orientaron a diferentes segmentos de mercado como el surf, el skate, las actividades outdoor y la ropa urbana. Durante de los años '90 y '00, estas empresas marcaron fuerte tendencia en el mercado de la indumentaria de la ciudad. Con la crisis del año 2001, como también ha sucedido en otras ciudades del país, los emprendedores comienzan a tomar iniciativas en el rubro de la indumentaria (Miguel, 2013). Hoy en día, el emprendedorismo asociado a este sector se manifiesta con firmeza, abocados a distintos segmentos de mercado.

Actualmente, el sector textil e indumentaria local se compone en un 55\% de empresas abocadas al tejido de punto y un $45 \%$ a la confección (Rech, 2019). Las firmas textiles son en su mayoría pequeñas y medianas empresas, de las cuales la mayor cantidad responde a estructuras familiares. Según informes, las firmas de esta rama representan alrededor del $13 \%$ de los locales industriales y el 10\% de la ocupación industrial de las PyME de la región (Observatorio PyME, 2009). El 13\% de las empresas textiles locales exportan, no obstante el monto exportado en 2007-8 representa en promedio sólo el 2\% de sus ventas. Por otro lado, también afirman que en 2008 , la utilización de la capacidad instalada alcanzaba el $72.6 \%$ del total. Cabe aclarar que tanto el sector textil como la sub-rama indumentaria padecen como patrón común la informalidad laboral, principalmente en los talleres a los cuales tercerizan, que muchas veces son de tipo domiciliario y unipersonal (Ruppel, 2019). Este factor afecta a las estadísticas del sector, ya que no pueden configurarse estadísticas fehacientes que expresen la realidad local. Otro dato importante para entender el sector es su modalidad de trabajo a fasón para marcas nacionales, principalmente de Buenos Aires y en el tejido de punto. Se estima que el $60 \%$ de la producción de sweaters nacional se realiza en Mar del Plata ${ }^{3}$. Esta dinámica ha dejado a un lado la producción para marca propia, porque los volúmenes más grandes son para otras empresas exigentes en términos de precios, calidad y tiempos de entrega. Se puede decir que en muchos casos, la marca propia queda en segundo plano por lo que se descuida un poco las cuestiones de branding e imagen de marca, comunicación del producto y tendencias (Monacchi, 2020). En el caso de la indumentaria, la producción se centraliza en los canales de comercialización propios, por lo que se evidencia una imagen de marca más consolidada y gestionada. 


\section{El diseño en el sector textil-indumentaria Mar del Plata}

Habiendo definido las características del sector y su historia, cabe preguntarse qué rol cumple el diseño en esta actividad económica local. Más aún, cuando nos referimos a un conglomerado PyME como es el caso de la industria textil-indumentaria marplatense, donde el diseño puede tener fuerte injerencia e impacto. Esta pregunta es válida ya que, en primer lugar y de forma más evidente, la industria textil está circunscripta al ámbito de la moda, lo cual la implica inevitablemente con el diseño. En segundo lugar, y a los fines de esta investigación el punto más interesante, tomando en cuenta la participación del diseño como factor estratégico de gestión dentro de la cadena de valor (Ivánez, 2000).

En términos de enseñanza de diseño, y particularmente aquellos vinculados al sector textil e indumentaria, se destaca la presencia de la Universidad Nacional de Mar del Plata, con su carrera específica de Diseño Industrial con orientación textil y orientación indumentaria. Por otro lado, la oferta educativa se nutre con el Instituto Palladio, con su carrera terciaria de Diseño y Producción de Indumentaria, el Instituto de Formación Profesional $\mathrm{N}^{\circ} 151$ con su Tecnicatura Superior en Industria Textil e Indumentaria y la Universidad Tecnológica Nacional con la Tecnicatura en Producción Textil. Por último, también aparece la figura de la Escuela de Capacitación Textil dependiente de la Cámara Textil, que hoy en día se encuentra cerrada. El lema de esta institución rezaba "Sin capacitación no hay industria" y fue creada en la década del '70, con el fin de enseñar oficios textiles para poder incorporarlos a las fábricas. La Escuela solía ser una institución modelo en la que los empresarios donaban materia prima y herramientas para poder capacitar futuros operarios y empleados. Sin embargo, la inestabilidad económica y la pérdida de fuerza de la Cámara provocaron que debiera cerrar sus puertas. Durante el 2015, volvió a reabrir y durante un año se dictaron cursos gratuitos variados. Con el cambio de gestión en la Cámara Textil, la Escuela cerró nuevamente.

Por otro lado, en la actualidad no se cuenta con datos precisos respecto a la participación de los diseñadores dentro de las empresas locales, pero sí se puede afirmar que su labor está orientada fuertemente a las cuestiones de producción. Esto podría vincularse al perfil empresarial fabril y técnico que manifiestan la mayoría de las empresas, principalmente las productoras de tejido de punto. Otro componente interesante que se visualiza en la rama indumentaria es el emprendedorismo. En este sentido, el aporte de la carrera de Diseño Industrial, particularmente en la UNMdP pero también en otras instituciones educativas superiores, es importante para la formación de emprendimientos. Estudios demuestran que esta carrera, fundada en 1994 en la UNMdP, es semillero emprendedor, destacando que el 65\% de sus graduados, emprenden o han emprendido alguna vez (Gennero de Rearte y otros, 2005). En el ámbito local, los emprendimientos del sector indumentaria y textil alcanzan el 27\% (Cabut y otros, 2019), ubicándose en el primer lugar, seguido por la categoría de Alimentos y bebidas (19\%) y Metalmecánica/Equipos electrónicos (10\%) Estos resultados podrían asociarse a las bajas barreras de entrada que poseen estas actividades, sumado a un aparato institucional (educativo, político, empresarial) que acompaña los procesos de desarrollo. En relación al sector textil e indumentaria, cabe destacar que está sumamente apuntalado por los nuevos canales de comercialización, como redes sociales, showrooms, asociación entre emprendedores, tiendas online, entre otras. 
Respecto a la asociatividad del sector, se debe resaltar la presencia de la Cámara Textil de Mar del Plata como primera institución que aglomeró a las empresas locales a partir de 1970. Sin embargo, esta organización fue perdiendo fuerza con las sucesivas crisis y recién en los últimos años volvió a posicionarse a través de las nuevas generaciones en los puestos dirigenciales de las firmas. Por otro lado, también aparece la Asociación de Confeccionistas de la Indumentaria y Afines (ACIA) que desde 2005 reúne empresas con marca propia, talleristas y proveedores de la industria. Actualmente brinda servicios tecnológicos y de capacitación para el sector. Si bien ambas instituciones nuclean a la gran mayoría de las empresas del sector, es cierto que sólo participan activamente algunas firmas ${ }^{4}$. Pero además, un dato importante para la actividad fue la conformación del Conglomerado TextilIndumentaria Mar del Plata Entramar, a fines de 2017 a través de un Programa de Apoyo a la Competitividad (PAC) para Micro, Pequeñas y Medianas Empresas, del Ministerio de Producción de la Nación. Esta entidad se manifestaba como superadora a las instituciones empresariales existentes (la Cámara Textil y la Asociación de Confeccionistas de la Indumentaria y Afines), ya que nucleaba no sólo a los socios de ambas, sino también a empresas no alineadas a ningún organismo, la Municipalidad y la Universidad Nacional. Este programa resulta una propuesta sumamente innovadora para el sector, que se manifestaba bastante desarticulado; además de la incorporación de nuevos actores a la mesa sectorial, como el gobierno y la academia (Monacchi y Canetti, 2018).

\section{Metodología adaptada al contexto, en búsqueda de una identidad corporativa}

El recorte de la población se realizó en base a este último acontecimiento, tomando como muestra el Conglomerado Textil Indumentaria Mar del Plata. La multiplicidad de actores de empresas de productos o servicios, instituciones y organizaciones da cuenta de una cadena de valor compleja e interesante de analizar. Sin embargo, esta diversidad de integrantes con sus respectivos intereses, presenta una dificultad para sentar a todos los participantes en la misma mesa. Esto no refiere a un ámbito problemático o de posturas enfrentadas entre los distintos integrantes, sino más bien por la imposibilidad de todos los participantes de coincidir y dedicar el mismo tiempo para la ejecución de las experiencias colaborativas. Además, cabe señalar que referimos a un sector productivo bastante tradicional, donde no son comunes este tipo de actividades que apelan al uso de capacidades creativas y sensibles, donde no hay cargos gerenciales definidos y muchas veces se concentran en el dueño de la empresa o sus hijos. Esta situación podría perfilar cierto escepticismo respecto a las herramientas de Design Thinking y sus posibles alcances. Es por ello que se decidió readaptar la metodología a las características del conglomerado local, para que la misma pudiera efectuarse en los términos necesarios y resultara efectiva a los fines de la investigación.

Otra cuestión fue la visualización de una posibilidad para enmarcar la actividad del Design Thinking, a partir de una necesidad puntual de las empresas del Conglomerado. La misma tuvo que ver con el diseño de su identidad corporativa como organización. Para 
ello, se estructuró una actividad en conjunto con el diseñador gráfico contratado para esta tarea con el objetivo de consensuar los distintos perfiles e intereses a través de la identidad corporativa. El Conglomerado se compone de actores diversos: micro, pequeñas y medianas empresas de diversa envergadura y trayectoria, algunas de ellas dedicadas al emblemático tejido de punto y otras a la indumentaria, algunas proveedoras de servicios y otras de producto en alguna de sus fases. Sumado a ello, la mesa sectorial también se conforma por actores académicos y gubernamentales. Es entonces, que la construcción del relato identitario, o también conocido como branding 5 , engloba mucho más que un objeto gráfico y exige el tomar conciencia de la estrategia a la cual se quiere apuntar como organización. Para el Conglomerado, estas cuestiones se constituían en la contratación de un diseñador gráfico para la identidad marcaria. Desde la perspectiva del diseño, era una oportunidad para trabajar con la creatividad y sensibilidad del grupo para construir en conjunto una identidad que albergara los intereses de todos. Para el diseño metodológico fue necesario mediar entre el objetivo general de la investigación y el objetivo de la actividad de branding propuesta para el Conglomerado:

En el caso de la investigación, su objetivo es incorporar herramientas de diseño a las experiencias de trabajo colaborativo y analizar la realidad del sector local.

En el caso del branding, su objetivo general será construir un concepto de identidad del conglomerado entre los distintos actores participantes.

La propuesta debería abordar ambos objetivos, priorizando que la actividad de branding pudiera lograr su finalidad para cumplir con la necesidad del sector. El objetivo de la investigación se abordaría de forma tangencial e implícita, integrada en las actividades orientadas a la construcción de la identidad colectiva. Para la ejecución de esta actividad se propusieron algunos interrogantes generales, como punto de partida para establecer qué información se deseaba obtener a través del trabajo de campo: ¿Cómo está compuesto el sector textil-confeccionista local? ¿Cómo se dan las relaciones dentro de esta cadena de valor? ¿Cuáles son las debilidades y fortalezas del sector? ¿Cuál es el valor diferencial del sector, y de los productos y servicios que ofrecen? ¿Cuál es la identidad de la industria textil-confeccionista local?

La metodología del Design Thinking es flexible y permite configuraciones diversas, dentro del abanico de herramientas que ofrece. Éstas son agrupadas en cinco etapas distintas, que responden a la lógica del pensamiento de diseño (Brown, 2009): empatizar, definir, idear, prototipar y evaluar. La cantidad de herramientas hacen que sea necesario seleccionar aquellas que son acordes a la actividad y a sus objetivos. En este caso, y por el tipo de industria tradicional con el cual se trabajaba, las premisas fueron: bajo/medio nivel de abstracción, actividades cortas y rápidas donde se pudieran visualizar resultados concretos. A partir de la selección de las herramientas, se realizó un cuadro de coherencia (Cuadro 1). Allí se exponen las preguntas devenidas de los objetivos de la investigación y la actividad de branding, correspondiendo a las variables, indicadores, herramientas a emplear, los patrones a tener en cuenta y la etapa del Design Thinking a la cual pertenecen. 


\begin{tabular}{|c|c|c|c|c|c|}
\hline Preguntas & Variables & Indicadores & Herramienta & $\begin{array}{l}\text { Patrones a tener en } \\
\text { cuenta }\end{array}$ & $\begin{array}{l}\text { Etapa } \\
\text { Design } \\
\text { Thinking }\end{array}$ \\
\hline \multirow{4}{*}{$\begin{array}{l}\text { ¿Cuáles son las } \\
\text { debilidades y } \\
\text { fortalezas del } \\
\text { sector? }\end{array}$} & \multirow[t]{4}{*}{ Contexto } & Fortalezas & \multirow{4}{*}{$\begin{array}{l}\text { Confección } \\
\text { de FODA en } \\
\text { el marco del } \\
\text { Focus Group } \\
\text { o Sesiones } \\
\text { Generativas }\end{array}$} & \multirow{4}{*}{$\begin{array}{l}\text { Ver qué puntos } \\
\text { determinan en común } \\
\text { y en cuáles discrepan. } \\
\text { ¿Hay factores internos y } \\
\text { externos que coinciden? }\end{array}$} & \multirow{4}{*}{$\begin{array}{c}\text { Empatizar } \\
/ \\
\text { Definir }\end{array}$} \\
\hline & & Debilidades & & & \\
\hline & & Oportunidades & & & \\
\hline & & Amenazas & & & \\
\hline \multirow{7}{*}{$\begin{array}{l}\text { ¿Cómo está } \\
\text { compuesto el } \\
\text { sector textil- } \\
\text { confeccionista } \\
\text { local? } \\
\text { ¿Cómo se dan } \\
\text { las relaciones } \\
\text { dentro de esta } \\
\text { cadena de valor? } \\
\text { ¿Qué se valora? }\end{array}$} & \multirow[t]{3}{*}{ Actores } & Cantidad & \multirow{3}{*}{$\begin{array}{l}\text { Diagrama de } \\
\text { afinidades } 0 \\
\text { relaciones }\end{array}$} & \multirow{3}{*}{$\begin{array}{l}\text { Comprender la propia } \\
\text { mirada respecto } \\
\text { del sector y sus } \\
\text { vinculaciones. Ver qué } \\
\text { actores consideran } \\
\text { importantes para } \\
\text { reforzar vínculo o } \\
\text { establecerlo. }\end{array}$} & \multirow{3}{*}{$\begin{array}{c}\text { Empatizar } \\
\text { / Definir }\end{array}$} \\
\hline & & Proximidad & & & \\
\hline & & $\begin{array}{l}\text { Actores directos/ } \\
\text { Indirectos }\end{array}$ & & & \\
\hline & \multirow[t]{2}{*}{ Rol del diseño } & $\begin{array}{l}\text { Cantidad de } \\
\text { diseñadores }\end{array}$ & \multirow[t]{2}{*}{$\begin{array}{l}\text { Termómetros } \\
\text { de calor }\end{array}$} & \multirow{2}{*}{$\begin{array}{l}\text { Identificar qué } \\
\text { importancia adjudican a } \\
\text { este valor }\end{array}$} & \multirow[t]{2}{*}{$\begin{array}{l}\text { Empatizar } \\
\text { / Definir }\end{array}$} \\
\hline & & $\begin{array}{l}\text { Consideración de } \\
\text { la importancia del } \\
\text { diseño }\end{array}$ & & & \\
\hline & \multirow[t]{2}{*}{ Sustentabilidad } & $\begin{array}{l}\text { Acciones } \\
\text { sustentables que } \\
\text { llevan a cabo }\end{array}$ & \multirow[t]{2}{*}{$\begin{array}{l}\text { Termómetros } \\
\text { de calor }\end{array}$} & \multirow[t]{2}{*}{$\begin{array}{l}\text { Identificar qué } \\
\text { importancia adjudican a } \\
\text { este valor }\end{array}$} & \multirow[t]{2}{*}{$\begin{array}{l}\text { Empatizar } \\
\text { / Definir }\end{array}$} \\
\hline & & $\begin{array}{l}\text { Consideración de } \\
\text { la importancia de } \\
\text { la sustentabilidad }\end{array}$ & & & \\
\hline \multirow[t]{2}{*}{$\begin{array}{l}\text { ¿Cuál es el valor } \\
\text { diferencial del } \\
\text { sector, y de los } \\
\text { productos y } \\
\text { servicios que } \\
\text { ofrecen? }\end{array}$} & $\begin{array}{c}\text { Tensiones } \\
\text { global/local }\end{array}$ & $\begin{array}{l}\text { Identificación de } \\
\text { la cadena de valor } \\
\text { y las tensiones } \\
\text { globales/locales }\end{array}$ & $\begin{array}{l}\text { Mapa de } \\
\text { localidad del } \\
\text { objeto-signo } \\
\text { y cadena de } \\
\text { valor }^{6} / \text { Mapa } \\
\text { de escenarios }\end{array}$ & $\begin{array}{l}\text { Comprender la cadena } \\
\text { de valor y sus relaciones } \\
\text { entre lo local, los regional } \\
\text { y lo internacional. ¿Cómo } \\
\text { identifican su producto } \\
\text { respecto de estos } \\
\text { valores? }\end{array}$ & Idear \\
\hline & $\begin{array}{l}\text { Modelo de } \\
\text { negocio }\end{array}$ & $\begin{array}{l}\text { Valor del negocio, } \\
\text { proveedores, } \\
\text { clientes, canales, } \\
\text { recursos, } \\
\text { actividades como } \\
\text { Conglomerado }\end{array}$ & $\begin{array}{l}\text { Matriz de } \\
\text { escenarios } \\
\text { futuros }^{8}\end{array}$ & $\begin{array}{l}\text { Establecer cuál es el } \\
\text { modelo de negocio como } \\
\text { Conglomerado. ¿Hay } \\
\text { discrepancias entre los } \\
\text { distintos participantes? } \\
\text { ¿Cómo pueden } \\
\text { visualizarlo a futuro } \\
\text { como Conglomerado? }\end{array}$ & Idear \\
\hline
\end{tabular}




\begin{tabular}{|l|l|l|l|l|l|}
\hline $\begin{array}{l}\text { ¿Cuál es la } \\
\text { identidad de la } \\
\text { industria textil- } \\
\text { confeccionista }\end{array}$ & \multirow{2}{*}{$\begin{array}{l}\text { Identidad } \\
\text { local? }\end{array}$} & \multirow{2}{*}{$\begin{array}{l}\text { Percepción de la } \\
\text { identidad }\end{array}$} & Brainstorming & $\begin{array}{l}\text { ¿Qué es la identidad? } \\
\text { ¿Qué implica? }\end{array}$ & \\
\cline { 3 - 4 } & & Storytelling & $\begin{array}{l}\text { ¿Qué queremos } \\
\text { representar de nuestra } \\
\text { identidad? }\end{array}$ & Prototipar \\
\cline { 4 - 5 } & & & $\begin{array}{l}\text { ¿Cómo haríamos para } \\
\text { contarlo? ¿A quién irá } \\
\text { dirigido? }\end{array}$ & \\
& & & & \\
& & & &
\end{tabular}

Cuadro 1. Cuadro de coherencia metodológica de la investigación. Fuente: Elaboración propia.

En este trabajo de campo, también se rescata el modelo de Investigación-Acción Participativa (Lewin, 1936) para definir el rol del diseño en la actividad. Este lineamiento metodológico aplica perfectamente para el caso de análisis y el enfoque del Design Thinking. Beatriz Galán (2007) adapta este modelo a la disciplina del diseño:

La investigación-acción participativa es una metodología que redefine la gestión de diseño. Es investigación en cuanto emplea métodos sistematizados para arribar a un diagnóstico. Es acción porque proyecta con las comunidades, de acuerdo con un criterio de cercanía vital, y, sobre todo, es participativa porque considera a las comunidades como sujetos y no como objetos de la investigación. La participación da sustentabilidad a la actividad proyectiva y la sustentabilidad, a su vez, se relaciona con la profundidad alcanzada por el conocimiento en el seno del proyecto o programa. La conciencia de los procesos que estructuran las redes de la cultura (p. 32).

Como expresa la autora, este modelo se enraíza en la participación comunitaria para la resolución de problemas de forma creativa y que propicien un sentimiento de pertenencia colectivo. De esta forma, la Investigación-Acción Participativa (IAP) funciona alternando procesos de reflexión teórica con instancias de aplicación práctica. Estos momentos se suceden consecutivamente: se reflexiona a través de conceptos teóricos para poder llevarlos a la práctica, y esas experiencias de campo se ponen en tela de observación y reflexión nuevamente, para posteriormente volver a verificarlas en la realidad. Estos procesos se podrían asemejar a la retroalimentación (iteración) que plantea el Design Thinking, como herramienta fundamental para la resolución de problemas y dinamismo de las soluciones. Asimismo, como plantea Galán, la visión del sujeto investigador reconoce que su aporte no sólo permite que se desarrollen las esferas productivas o comunidades, sino que también retroalimenta la investigación perfilando a nuevos interrogantes para futuros proyectos. Además, resalta la importancia del análisis crítico de las experiencias de transferencia, 
como también da lugar a teorizar sobre posibles modelos de análisis para comprender el impacto que genera el diseño en los contextos locales (Galán en Ruppel, 2019).

Evidentemente, el IAP, el Design Thinking y el tema de investigación que se desarrolla en este trabajo poseen múltiples puntos de conexión. Es más, a partir de la postura que plantea Galán, del rol del diseño con las comunidades locales y el valor de la experiencia práctica para fortalecer soluciones genuinas, ayuda a contrarrestar las críticas corporativistas del Design Thinking (Kimbell, 2011). Es posible que las teorías hegemónicas de este enfoque prevalezcan en los países desarrollados, pero eso no implica que el pensamiento de diseño con herramientas específicas, no puedan ser accesibles para las realidades locales.

\section{Resultados de la construcción colectiva}

La propuesta de Design Thinking para el Conglomerado Textil Indumentaria Mar del Plata se ejecutó en dos encuentros 9 . Como se mencionó anteriormente, estos encuentros se presentaron como una actividad de branding y se trabajó junto con el diseñador gráfico encargado de la realización del logo institucional. De forma conjunta, se estructuró la actividad y se llevó a cabo.

Los participantes, en su mayoría, eran empresarios y dentro del Conglomerado estaban a cargo de las cuestiones vinculadas a marketing y la generación de una marca ciudad. También se sumaron representantes directivos del Conglomerado, de la Universidad Nacional de Mar del Plata y de la Municipalidad de General Pueyrredón ${ }^{10}$. Entre los dos encuentros, aunque se forma alternativa, participaron 14 personas.

El relevamiento de la actividad se hizo mediante grabación de audio y video y registro fotográfico. Durante el primer encuentro, se realizaron sólo las actividades de Focus Group y Diagrama de Afinidades. Durante el segundo encuentro, se completaron los Termómetros de calor, el Mapa de localidad objeto/signo y cadena de valor, Brainstorming y Storytelling. La herramienta de Mapa de escenarios y Matriz de escenarios futuros finalmente fueron relegadas, por el tiempo que consumían las actividades y por el nivel de abstracción que suponían. Se alternaron actividades grupales con otras individuales, pero todas de forma anónima. En las imágenes 1, 2, 3 y 4 pueden evidenciarse los resultados de la experiencia de trabajo colaborativo. 

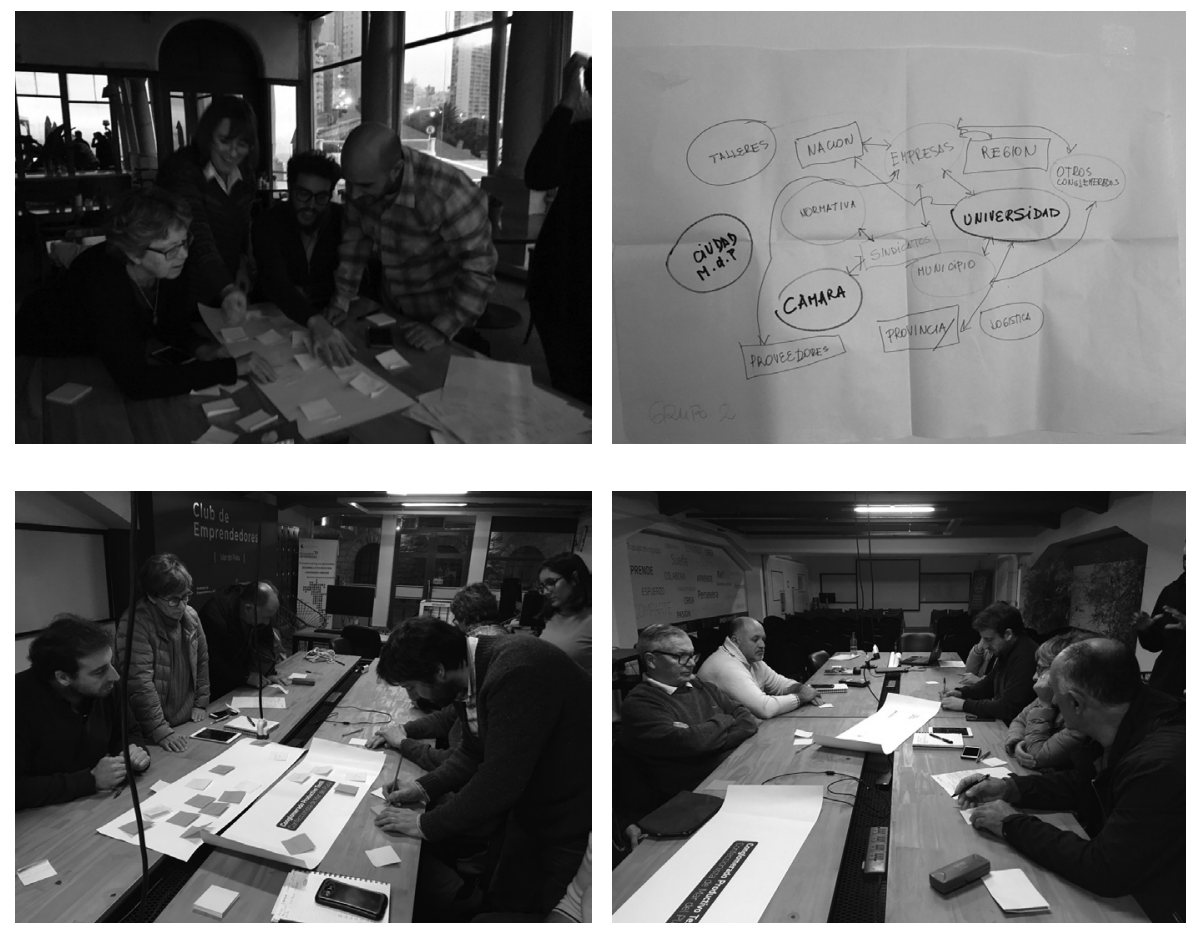

Imágenes 1, 2, 3 y 4 (izq. a der., de arriba a abajo). Resultados de la actividad colaborativa con participantes del Conglomerado Textil Indumentaria Mar del Plata. Junio 2019. Fuente: Elaboración propia.

El Cuadro 2 muestra los resultados emergentes de la actividad colaborativa:

\begin{tabular}{|c|c|c|}
\hline Preguntas & Herramienta & Resultados \\
\hline $\begin{array}{l}\text { ¿Cuáles son las } \\
\text { debilidades y } \\
\text { fortalezas del sector? }\end{array}$ & Focus Group & $\begin{array}{l}\text { Positivo: Producción local - industria local marplatense / } \\
\text { Trabajo en unidad / Mantenerse vigente con el producto - buscar } \\
\text { oportunidades / ecosistema - heterogeneidad. } \\
\text { Negativo: Conservador / Estático / Economía recesiva } \\
\text { Indefinido: Historia, tradición, trayectoria / Crisis, complejidad }\end{array}$ \\
\hline
\end{tabular}

continúa $>>$ 


\begin{tabular}{|c|c|c|}
\hline \multirow[t]{2}{*}{$\begin{array}{l}\text { ¿Cómo está } \\
\text { compuesto el sector } \\
\text { textil-confeccionista } \\
\text { local? } \\
\text { ¿Cómo se dan las } \\
\text { relaciones dentro de } \\
\text { esta cadena de valor? } \\
\text { ¿Qué se valora? }\end{array}$} & $\begin{array}{l}\text { Diagrama de } \\
\text { afinidades } 0 \\
\text { relaciones }\end{array}$ & $\begin{array}{l}\text { Actores actuales: Universidad, Gobierno (local/nacional), } \\
\text { Empresas (a través de las cámaras), Proveedores, Talleres, } \\
\text { Sindicatos, Clientes } \\
\text { Actores potenciales: Asociación de Tecnologías de la Información } \\
\text { y la Comunicación de Mar del Plata y zona (ATICMA), Prensa, } \\
\text { Talleres, Otros conglomerados, Unión del Comercio, la Industria y } \\
\text { la Producción (UCIP) }\end{array}$ \\
\hline & $\begin{array}{l}\text { Termómetros de } \\
\text { calor }\end{array}$ & $\begin{array}{lcc} & \text { EVALUACIÓN }^{\text {PONDERACIÓN (a futuro) }}{ }^{11} \\
\text { SUSTENTABILIDAD } & 4 & 2 \\
\text { DISEÑNO } & 2 & 4 \\
\text { PRODUCCIÓN LOCAL } & 1 & 3 \\
\text { INNOVACIÓN } & 3 & 2 \\
\text { TECNOLOGÍA } & 1 & 1\end{array}$ \\
\hline \multirow[t]{2}{*}{$\begin{array}{l}\text { ¿Cuál es el valor } \\
\text { diferencial del sector, } \\
\text { y de los productos y } \\
\text { servicios que ofrecen? }\end{array}$} & $\begin{array}{l}\text { Mapa de } \\
\text { localidad del } \\
\text { objeto-signo } \\
\text { y cadena de } \\
\text { valor / Mapa de } \\
\text { escenarios }\end{array}$ & $\begin{array}{l}\text { Mapa de localidad del objeto-signo: Marcado énfasis global en las } \\
\text { cuestiones referidas a Forma/función, Tecnología y Materias primas } \\
\text { y la Continuidad. Raíz histórica tiene una definida prioridad local. } \\
\text { Cadena de valor: Fuerte presencia de proveedores nacionales y una } \\
\text { preminencia de los productores en términos locales. La distribución, } \\
\text { los canales de difusión y la comercialización tienen tendencia al } \\
\text { ámbito nacional, aunque con presencia local. Consumidores } \\
\text { también se centralizan en el territorio nacional, aunque un solo } \\
\text { participante manifestó incursionar en el mercado global. Se puede } \\
\text { observar claramente en el cuadro una preponderancia en el área } \\
\text { nacional en la gran mayoría de las etapas de la cadena. }\end{array}$ \\
\hline & $\begin{array}{l}\text { Matriz de } \\
\text { escenarios } \\
\text { futuros }\end{array}$ & No se realizó \\
\hline \multirow[t]{2}{*}{$\begin{array}{l}\text { ¿Cuál es la identidad } \\
\text { de la industria textil- } \\
\text { confeccionista local? }\end{array}$} & Brainstorming & $\begin{array}{l}\text { Tópicos referidos a la asociatividad, como "fuerza", "unión", } \\
\text { "asociatividad", "sinergia", "construcción", "compartir aspectos } \\
\text { comunes". Palabras vinculadas a la identidad de la industria como } \\
\text { "arraigo", "pertenencia", "historia", "trayectoria y proyección". } \\
\text { "Calidad" y "diseño", "singuralidad" y "diferenciación" del producto } \\
\text { local. }\end{array}$ \\
\hline & Storytelling & $\begin{array}{l}\text { Nombres surgidos de la narrativa. "Olas Tejidas", "Tejido y sal", } \\
\text { "Mar tejido", "Unitex", "Po. Tex.Mar, "Entramar" }\end{array}$ \\
\hline
\end{tabular}

Cuadro 2. Resultados de las herramientas utilizadas en la actividad colaborativa. Fuente: Elaboración propia. 


\section{Percepciones de la experiencia colaborativa}

La propuesta de implementar herramientas del Design Thinking en un contexto local se visualizaba compleja al referir a un sector industrial bastante conservador y tradicional, con un marcado perfil técnico. Sin embargo, el trabajo de campo se consideró positivo por la cantidad de información cualitativa recabada y la posibilidad de interactuar de primera mano con el sector textil y de indumentaria marplatense. El Conglomerado propició un espacio de encuentro y acción donde el diseño podía intervenir, proponiendo actividades sensibles y creativas que favorecieran el trabajo en equipo y la formulación de ideas colectivas. Por ese lado, la metodología colaboró en los procesos de integración, tanto de las empresas, como del resto de los actores participantes en el Conglomerado. Asimismo, al haber sido contratado un profesional de diseño gráfico, también se lo incorporó a la actividad de Design Thinking, generando nuevos canales de comunicación e información entre colegas. La instancia interdisciplinaria que plantea esta metodología fue lograda, como así también la pluralidad y participación de todos los integrantes.

Respecto a la metodología propiamente y la estructuración de la actividad, devienen algunas cuestiones: Complejidad para entender ciertas consignas o premisas, principalmente cuando se refería a esquemas predeterminados (Termómetros, Mapas de localidad). Las explicaciones debían ser sumamente concisas y en algunos casos no se resolvieron de forma completa. Alto nivel de abstracción en algunas actividades. Resultaba complejo para los participantes entender hacia dónde llevaba la actividad cuando los resultados no eran concretos, y eso producía cierto aburrimiento o incomprensión. Necesidad de suprimir algunas actividades planteadas. En el transcurso de los encuentros, las percepciones antes mencionadas, hicieron que se decidiera anular tres actividades que ya estaban pautadas. Esto tuvo que ver con la extensión de los encuentros y los niveles de atención que manejaban los participantes. Rigor de los tiempos, punto que remarca la metodología y cuya gestión se hace muy difícil durante las actividades. La organización previa de las actividades fue fundamental para un desarrollo ordenado y con los elementos necesarios. También el espacio físico donde se desarrolla la actividad resulta importante en términos de comodidad, esparcimiento y comunicación. Flexibilidad en las actividades para evitar el agotamiento de los participantes. Si bien estaban pautadas y organizadas, se fueron amoldando a la percepción del moderador respecto del equipo y su participación.

Por parte de los participantes: Dificultad para consensuar la participación constante de todos los integrantes, entre los dos encuentros, y dentro del mismo encuentro. Esto implicó que no hubiera un número constante de participantes, sino que algunos estuvieron presentes en uno de los encuentros, pero no en el siguiente. De la misma forma, algunos participaron sólo un rato y no el encuentro completo. Necesidad de visualizar los resultados de la actividad y dificultad para manejar niveles de abstracción. Estas cuestiones se traducen en momentos de aburrimiento, distracción o participación escasa de algunos participantes. Se observa la necesidad de palpar materialmente lo que se está construyendo a nivel conceptual; es posible que esta capacidad proyectual del diseño no se perciba de la misma forma por todas las personas. Respeto, atención unánime y participación grupal integrada. En todas las actividades se reflejaron estos aspectos, tanto dentro del grupo, como con los moderadores. De la misma forma que se ejecutaron todas las actividades 
propuestas, por más que no quedaba muy en claro cuál era la finalidad. Satisfacción con la actividad y los resultados obtenidos. Es posible que evidenciar el trabajo invertido en un producto concreto (sea un nombre, un logo, etc.), produzca satisfacción y tranquilidad.

\section{Discusiones}

Esta experiencia colaborativa con distintos actores de la cadena de valor textil-indumentaria de Mar del Plata, ha devenido en algunas reflexiones respecto al rol del diseño en los territorios y la implementación de metodologías proyectuales. Por un lado, se reafirma la importancia del diseño y la gestión de las capacidades de innovación en los escenarios económicos locales, principalmente en los países en vías que desarrollo que distan de los modelos centralistas. La competitividad del mercado exige que las pequeñas y medianas empresas e industrias generen diferenciales de valor en sus productos y servicios. Pardo Kuklinski (2014) resalta que las competencias creativas se vuelven esenciales para moverse en la economía digital global, como así también para generar estrategias innovadoras. De la misma forma, adquieren relevancia la interdisciplinariedad (lo que el autor denomina la construcción de un "arquetipo exótico"), el trabajo en equipo y la construcción de redes como potenciadores de innovación. En este sentido, la pequeña y mediana empresa emerge como espacio de intercambio y canalizador de tendencias globales, que deben readecuar a sus propias idiosincrasias.

Los contextos periféricos absorben los patrones y las dinámicas de consumo, pero sin el correlato local de las estructuras sistémicas de factores geográficos, el desarrollo de los mercados, de los sistemas de financiamiento, de los ecosistemas que puedan sostener y anidar patrones de consumo y producción. (...) De allí que los sistemas de la pequeña escala hayan sido revalorizados como sistemas alternativos, (...) permitiendo que la producción en pequeña escala sea un laboratorio de interacciones sociales, económicas y tecnológicas del sistema productivo... (Galán, 2007).

En síntesis, el diseño asume un compromiso particular en el territorio como factor de innovación, potenciado en el ámbito de las PyMEs.

En el caso de esta experiencia, y siguiendo las lógicas de desarrollo local que plantean Arocena (2002) y Vázquez Barquero (2000), fue la situación de crisis que atravesaba el sector el puntapié para trabajar en conjunto. Esto es destacable, ya que no hay registro de acciones colaborativas previas dentro de la trayectoria textil-indumentaria local. Los autores refieren a que la vulnerabilidad de la crisis, hace aflorar el sentimiento de salvar a la región y motiva a las sociedades a accionar. Estas cuestiones apuntalan a un sentimiento compartido y consolidan espacios de trabajo conjunto, superando las rispideces y competencias. En este camino, el rol de las instituciones es importante para construir entornos territoriales facilitadores a través de las interacciones público-privadas. Las fórmulas y soluciones centralistas no son respuesta a las demandas de los territorios; la construc- 
ción comunitaria es la que refleja un consenso de realidades, y embandera a los actores a trabajar por ella. Sumado a esto, "la inteligencia en contexto" (Galán, 2019) del diseño, manifestada en la comprensión y lectura del territorio, fue en primera instancia detectar necesidades puntuales del Conglomerado donde intervenir y colaborar con los procesos. Esta visión de la oportunidad, no se reduce solamente un acto casual, sino que surge de la participación en las mesas sectoriales, empatizar con el resto de los actores, dar a conocer el trabajo desde la disciplina. El trabajo colectivo exige un proceso de involucramiento con la situación y entendimiento de la problemática. La lógica de Investigación-Acción debe observar y aprender del territorio para luego poder interactuar con él.

Respecto a la aplicación de herramientas de diseño en la experiencia colaborativa, se pueden mencionar algunos aspectos conclusivos. En primer lugar, se puede afirmar que el Design Thinking o pensamiento de diseño propone un marco de contención metodológico que adquiere sentido y validez únicamente en la interacción y aporte entre diferentes personas. La riqueza está en la sinergia participativa que se genera, el debate, el cruce de opiniones, los consensos; en definitiva, la creación de valor compartido (Manzini, 2015; Porter y Kramer, 2011). Sin embargo, en términos investigativos, este perfil dificulta una recolección estructurada de datos y su posterior procesamiento. Además, la variedad de visiones sobre el pensamiento de diseño dificulta el estudio empírico y su incorporación dentro de las estructuras organizacionales (Kimbell, 2011). Las pequeñas y medianas empresas locales, por su perfil tradicional, no están acostumbradas a las actividades lúdicas y creativas y en casos, se conciben como pérdidas de tiempo. Sin embargo, cuando comienzan a percibir los resultados tangibles del proceso, la perspectiva cambia. Es importante sensibilizar sobre estas experiencias, tanto en forma sectorial, como al interior de las organizaciones, porque permiten captar elementos que de otra manera no se alcanzarían. La construcción colaborativa de la mano del diseño, abre puertas a lo inesperado, lo espontáneo e impredecible, que puede ser fermento de futuras innovaciones.

El contexto de pandemia que se vive en la actualidad ayuda a repensar prospectivamente las experiencias colaborativas en el marco del diseño; más aún en torno a las organizaciones de carácter local. Si bien es difícil establecer conclusiones cuando aún estamos atravesando este fenómeno, se pueden hacer ciertas lecturas respecto a lo ocurrido y pensar estrategias que potencien el valor local-global. En primer lugar, el fortalecimiento de los esquemas territoriales a partir de la revalorización del rol de las empresas, profesionales e instituciones en este marco de pandemia. No sólo como proveedores de insumos y servicios, sino también como partícipes en acciones colaborativas (en el caso textil local, la confección de indumentaria sanitaria por parte de las empresas y la unión de profesionales del diseño, quienes normalizaron diseños de libre acceso para confeccionar de forma hogareña de protección básica como mascarillas, barbijos, etc.). Estos vínculos generados como consecuencia de la emergencia sanitaria, demuestran la potencialidad de la acción colectiva y su importancia para el territorio local. De la misma forma, los usuarios adquieren un nuevo rol activo en esta problemática; ya no son únicamente consumidores sino que se involucran desde sus hogares, por ejemplo, a partir de la producción casera o de la compra por adelantado para ayudar a los emprendedores locales. Además, la relevancia y masividad absoluta que ha adquirido el uso de redes sociales y de nuevas plataformas online, es otro factor importante para los tiempos que se avecinan. Si bien el uso de Internet 
ya estaba sumamente extendido, en los últimos tiempos se ha convertido indispensable en la vida cotidiana. Todas estas relaciones nuevas o diferentes, totalmente espontáneas y surgidas de la coyuntura particular, pueden ser el puntapié para crear nuevos espacios de trabajo en conjunto entre empresas, instituciones, consumidores y gobierno donde el diseño tiene mucho para aportar y acompañar.

A modo de reflexión abierta -porque en el contexto actual aún hay más dudas que certezas- se puede decir que el diseño dentro del territorio puede actuar como facilitador de procesos de adaptación al cambio tecnológico (Thomas, 2008) por parte de la comunidad. La apropiación colectiva de las tecnologías le da nuevos significados y usos, y condiciona que funcionen o no en un territorio. Con el caso de las herramientas de diseño para las organizaciones, sucede lo mismo. Es necesario entender las dinámicas del lugar (su cultura, su historia, sus diferencias y problemáticas) para poder trabajar con y para ellos. El contexto de emergencia sanitaria no hace más que reafirmar estas cuestiones y plantear nuevos interrogantes para seguir trabajando. Las zonas flexibles de trabajo colectivo son claramente nichos de innovación para el territorio y es allí donde el diseño puede alcanzar un enfoque estratégico que excede ampliamente el mundo objetual.

\section{Notas}

1. La abreviación PyME, refiere a pequeñas y medianas empresas. Según la clasificación Secretaría de la Pequeña y Mediana Empresa y Desarrollo Regional, se considera microempresas aquellas que emplean menos de 15 personas; pequeña entre 15 y 60 empleados y mediana de 60 a 235 empleados.

2. Página web de la institución, en línea: https://www.entramar.net.ar/

3. Declaraciones de representantes directivos de la Cámara Textil de Mar del Plata, año 2015. En línea: http://www.telam.com.ar/notas/201507/111584-industria-textil-mar-delplata-lana.php

4. Esto se ha podido evidenciar en el trabajo de campo realizado con el Conglomerado Textil Indumentaria Mar del Plata Entramar durante el 2019 y ya había sido manifestado por varios informantes calificados.

5. Se entiende que las organizaciones deben establecer una identidad corporativa fuerte, coherente y distintiva y comunicarla adecuadamente a su público (Capriotti, 2009). Branding hace referencia al proceso de hacer y construir una marca administrando y gestionando los activos que identifican a la misma y que influyen en su valor (Gaitán, 2007). Esta construcción define la identidad o esencia de la organización.

6. Mapa de localidad del objeto/signo extraído de Cervini, A; Kayser, J. (2004) Identidad Estratégica. Alternativas locales en mercados globales, con modificaciones propias para la actividad.

7. Ídem anterior

8. Matriz de escenarios futuros. extraído de Castillo Cabezas, Javier. Seminario Empresas Innovadoras, Maestría en Diseño orientada a la estrategia y la Gestión de la innovación, UNNOBA - UNMdP. 
9. Los dos encuentros tuvieron lugar el 6 y el 12 de Junio del 2019, en el Club de Emprendedores del Torreón del Monje, Mar del Plata, Argentina.

10. Como representantes del Conglomerado, Juan José Cruz, coordinador y Nahuel Ares Rossi, administrativo; desde la UNMdP, Silvia Stivale, Rocío Canetti y Guillermo Bengoa, del CIPADI, y Fernando Graña, de la FCEyS; por parte de la MGP, Alicia Meschini. Empresarios representantes de la Comisión 1: Gustavo Duc (Eva Mujer), Nicolás Leoz (Deixe) y Rubén Cedrón, por ACIA; Lorena Cageao (Bordatex/Baby Party); Guillermo Fasano (Inés Meyer); Fabián Basualdo (Promotextil); y Gabriela Ezpeleta (Del Cerro).

11. En este ítem se muestran los resultados promedio entre las respuestas de todos los participantes. La evaluación determinaba en qué instancia, según la mirada del participante, se encuentra hoy en Conglomerado y la ponderación permitía visualizar cuán importante se considera el tema analizado para tener en agenda de trabajo a futuro. Para la explicación en este cuadro, se numera el 1 como mejor posicionado/más importante y 4 , como menos.

\section{Listado de referencias bibliográficas}

Arocena, J. (2002). El desarrollo local: un desafío contemporáneo. Uruguay: Taurus -Universidad Católica.

Brown, T. (2009). Change by Design. How Design Thinking transforms organizations and inspires innovation. HarperCollins e-books.

Brown, T. (2008). Design Thinking. Harvard Business Review América Latina.

Buchanan, R. (1992). Wicked Problems in Design Thinking. MIT Press. Design Issues, Vol. 8, No. 2, (Spring, 1992), pp. 5-21.

Cabut, M; Mocerla, O. A; Bertone, B; y Petrillo, J. D. (2019). Estudio sobre las incubadoras de la Universidad Nacional de Mar del Plata y de la Unión del Comercio, la Industria y la Producción. Observatorio Tecnológico - OTEC. Departamento de Ingeniería Industrial, Facultad de Ingeniería, UNMdP. Argentina.

Favero, B. (2011). Las tramas de una identidad: el trabajo textil doméstico entre las mujeres inmigrantes italianas de posguerra en Mar del Plata a partir de entrevistas orales. Jornadas Internacionales Sociedad, Estado y Universidad. Mar del Plata, Argentina.

Gaitán, R. (2007). Branding para primíparos. Disponible en: http://logotrend.blogspot.com. ar/2007/10/brandingpara-primparos-por-ricardo.html

Galán, B. (2018). Reconstruyendo el entramado de una sociedad creativa. Estrategias para la formación de diseñadores en contextos de complejidad. Centro de Estudios en Diseño y Comunicación. Cuaderno 67 | pp 63-100. Universidad de Palermo, Argentina.

Galán, B. (compiladora) (2011). Diseño, proyecto y desarrollo. Miradas del período 2007-2010 en Argentina y Latinoamérica. Wolkowicz Editores.

Galán, B. (2007). Diseño, Academia y Comunidad: Transferencia de Diseño en comunidades productivas emergentes. En Diseño y Territorio. Bogotá: UNC.

Garbarini, R. (2019). Seminario Procesos y Estrategias Innovadores del Diseño junto a la docente González Insúa, M. en el marco de la Maestría en Diseño orientada a la Estrategia y la Gestión de la Innovación, dependiente de la UNNOBA, en su cohorte 2018 - UNMdP. 
Gennero de Rearte, A.; Graña, F; Liseras, N. y otros (2009). Informe regional. Industria manufacturera. Evolución reciente, situación actual y expectativas de las PyMES industriales. Observatorio PyME Regional General Pueyrredón y zona de influencia de la Provincia de Buenos Aires. Universidad Nacional de Mar del Plata, Argentina.

Gennero de Rearte, A. y otros (2008). Conductas innovativas de las firmas en aglomeraciones productivas del sector textil-confecciones. XIII Reunión Anual de la Red Pymes Mercosur. Universidad Nacional de General San Martín, Argentina.

Gennero de Rearte, A. M; Liseras, N; Baltar, F y Graña, F (2005). Creación de empresas por graduados universitarios. Comunicación presentada en X Reunión Anual de la Red PyMEs-MERCOSUR, Neuquén, Argentina.

Gennero de Rearte, A. (1991). Los distritos industriales como modelo de organización industrial: el caso del tejido de punto marplatense. CFI/CEPAL, Documento de trabajo No 25. Mar del Plata, Argentina.

Iváñez Gimeno, J. (2000). La gestión del diseño en la empresa. McGraw-Hill.

Kimbell, L. (2011). Rethinking Design Thinking: Part I. Journal Design and Culture. Volume 3, 2011 - Issue 3. P. 285-306.

Lewin, K. (1936). Principios de la Psicología Topológica. Nueva York: McGraw-Hill.

Martin, R. (2009). The design of business: Why design thinking is the next competitive advantage. Cambridge: Harvard Business Press.

Manzini, E. (2015). Cuando todos diseñan. Una introducción al diseño para la innovación social. Experimenta Theoria.

Miguel, P. (2013). Emprendedores del Diseño: Aportes para una sociología de la moda. Buenos Aires: Eudeba.

Monacchi, M. C. (2020). Diagnóstico de la cadena de valor textil marplatense a partir de la herramienta analitica del Design Thinking. Informe Final Beca A 2017-2020. Universidad Nacional de Mar del Plata. Aún no publicado.

Monacchi, M. C. y Canetti, R. (2018). Teorizando el mapa del diseño. Caso CeDiS, Mar del Plata, Argentina. Ecuador: Revista Daya. Diseño, Arte y Arquitectura. Número 5, Junio 2018 - Diciembre 2018, pp. 87 - 101.

Monacchi, M. C. (2017). Destejiendo historias y recuperando la identidad del tejido de punto marplatense. Mar del Plata: Revista I+A: Investigación + Acción I, Año 20, No 19, págs. 61-78.

Pardo Kuklinski, H. (2014). Opportunity Valley. Lecciones <aún> no aprendidas de treinta años de contracultura digital. Barcelona: Outliers School.

Porter, M y Kramer, M. (2011). La creación de valor compartido. Harvard Business Review América Latina.

Rech, L. (2019). Exposición Estudio global de las empresas del sector textil y confecciones Mar del Plata-Batán. Grupo de Análisis Industrial. Centro de Investigaciones Económicas y Sociales, FCEyS, UNMdP. Mar del Plata, Argentina: Torreón del Monje.

Rowe, P. (1987). Design thinking. England: MIT Press.

Ruppel, C. (2019). Innovación social aplicada a las pequeñas esferas productivas: Caso de las costureras a domicilio en la región de Mar de Plata. Tesis de maestría en Diseño orientada a la estrategia y la gestión de la innovación, dependiente de la UNNOBA.

Simon, H. (1969). The sciences of Artificial. London: MIT Press. 
Thomas, H. (2008). Estructuras cerradas versus procesos dinámicos: trayectorias y estilos de innovación y cambio tecnológico. En Thomas, $\mathrm{H}$ y Buch, A. (coordinadores) Actos, actores y artefactos. Sociología de la tecnología. Quilmes: Ciencia, Tecnología y Sociedad. Bernal, Universidad Nacional de Quilmes.

Vianna, M. y otros (2016). Design Thinking, innovación en los negocios. Rio de Janeiro: MJV Press.

Vázquez Barquero, A. (2000). Desarrollo endógeno y globalización. Revista EURE v.26 n.79 diciembre 2000, Santiago de Chile.

\begin{abstract}
The design expounds on the flexible boundary of the community adaptation to technological changes. This premise would explain why standardized solutions don't work at the same way in different contexts. In this process, the design could contribute as an adaptation's interface. This article describes a collaborative experience with different actors of clothing-and-textile value chain from Mar del Plata, Argentina in 2019. In a complex context, the enterprises, the government and the academic institutions form a local cluster. The design proposal is to accompany the cluster's identity process including design thinking tools. The results of this experience aim to demonstrate the power of network as the core of local innovations.
\end{abstract}

Keywords: Collaborative design - Design Thinking - Local cluster - Clothing-and-textile value chain.

Resumo: $\mathrm{O}$ design expõe os limites flexíveis da adaptação da comunidade às mudanças tecnológicas. E assim que as soluções padronizadas não funcionam do mesmo jeito em diferentes contextos e o design pode contribuir como uma interface de adaptação. Este artigo descreve uma experiência colaborativa com diferentes atores da cadeia de valor de vestuário e têxtil de Mar del Plata, Argentina, em 2019. Em um contexto complexo, as empresas, o governo e as instituições acadêmicas formam um cluster local. A proposta de design é acompanhar o processo de identidade do cluster, incluindo ferramentas de design thinking. Os resultados dessa experiência procuram demonstrar o poder da rede como o núcleo das inovações locais.

Palavras chave: Design colaborativo - Design Thinking - Cluster local - Cadeia de valor de vestuario e têxtil.

[Las traducciones de los abstracts fueron supervisadas por el autor de cada artículo] 